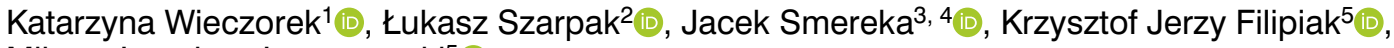
Miłosz Jarosław Jaguszewski ${ }^{5}$

${ }^{1}$ Student's Journal Club, Lazarski University, Warsaw, Poland

${ }^{2}$ Comprehensive Cancer Center in Bialystok, Bialystok, Poland

${ }^{3}$ Polish Society of Disaster Medicine, Warsaw, Poland

${ }^{4}$ Department of Emergency Medical Service, Wroclaw Medical University, Wroclaw, Poland

$51^{\text {st }}$ Department of Cardiology, Medical University of Gdansk, Gdansk, Poland

\title{
Should we resuscitate COVID-19 patients with non-shockable rhythms?
}

As of May 25, 2020, there were reported $5,432,512$ confirmed COVID-19 cases, with the mortality rate of nearly $6.9 \%$. So far, no data have been available on the survival of patients with COVID-19 after cardiac arrest. Currently, a study by Shao et al. ${ }^{1}$ is the only one to present the outcomes of in-hospital cardiac arrest (IHCA) among patients with COVID-19 pneumonia. Shao et al. indicate that the return of spontaneous circulation after IHCA is observed in $13.6 \%$ of patients with severe COVID-19 pneumonia, and the 30-day survival is even worse and equals $2.9 \%$. Owing to the progressive virus pneumonia in these patients, they should be monitored and treated in ICUs, as this increases their statistical survival compared with general wards $(\mathrm{RR}=14.74 ; 95 \% \mathrm{Cl}: 1.6-135.48 ; \mathrm{p}=0.02)$.

The prevailing pandemic forces us, as medical professionals, to accept compromises, which often includes making difficult choices about whom to save. Hence, the question arises whether we should resuscitate all patients with COVID-19. The answer may contradict the general principle of "first do not harm" and pose ethical dilemmas. We must remember that in addition to the safety of a given patient, we must also take into account our safety, as well as that of other patients. A COVID-19 patient resuscitation increases the risk of infection among medical personnel and subsequent transmission of the virus to other patients or family members. Moreover, in times of resource insufficiency, medical personnel contamination and the consequent quarantine further reduce the potential of hospitals. Shao et al. [1] revealed that the highest 30-day survival rate was observed in patients with VF/pVT rather than PEA or asystole as the initial rhythm $(\mathrm{RR}=48.00 ; 95 \% \mathrm{Cl}: 4.61-411.04 ; \mathrm{p}<0.001)$.
Another dilemma associated with the resuscitation process is that, according to the Centers for Disease Control and Prevention, each aerosolizing procedure should be performed with personal protective equipment, consisting of N95 respirators, eye protection, gloves, and gowns; the procedures themselves should be implemented in airborne infection isolation rooms [2]. In severe SARSCoV-2 pneumonia, the main cause of cardiac arrest is respiratory failure and the most frequently observed rhythm is asystole resulting from hypoxia. Intubation and ventilation of COVID-19 patients have poor survival rates [3].

In summary, in order to reduce the risk of infection, we should consider not resuscitating COVID-19 patients with observed non-shockable rhythms, especially those over 60 years of age.

\section{Conflict of interest}

The authors declared no conflict of interest.

\section{References}

1. Shao F, Xu S, Ma X, et al. In-hospital cardiac arrest outcomes among patients with COVID-19 pneumonia in Wuhan, China. Resuscitation. 2020; 151: 18-23, doi: 10.1016/j.resuscitation.2020.04.005, indexed in Pubmed: 32283117

2. Centers for Disease Control and Prevention. Interim Infection Prevention and Control Recommendations for Patients with Suspected or Confirmed Coronavirus Disease 2019 (COVID-19) in Healthcare Settings. https://www.cdc.gov/coronavirus/2019-ncov/hcp/infection-control-recommendations.html (18.04.2020).

3. Yang $X, Y u Y, X u J$, et al. Clinical course and outcomes of critically ill patients with SARS-CoV-2 pneumonia in Wuhan, China: a single-centered, retrospective, observational study. Lancet Respir Med. 2020; 8(5): 475-481, doi: 10.1016/S2213-2600(20)30079-5, indexed in Pubmed: 32105632 